\title{
Tocilizumab exerts anti-inflammatory activity in six critically ill COVID-19 patients: a retrospective analysis
}

\author{
Shen Qian ${ }^{1,2 \#}$, Jianghong An ${ }^{3 \#}$, Furong $\mathrm{Qi}^{2 \#}$, Ling $\mathrm{Ye}^{4}$, Qiong Chen ${ }^{3}$, Xunqi Liu ${ }^{3}$, Lixin Xie ${ }^{1}$, Guobao $\mathrm{Li}^{2}$ \\ ${ }^{1}$ College of Pulmonary and Critical Care Medicine, Chinese PLA General Hospital, Beijing, China; ${ }^{2}$ Department of the Third Pulmonary Disease, \\ The Third People's Hospital of Shenzhen/National Clinical Research Center for Infectious Diseases, Shenzhen, China; ${ }^{3}$ Department of Oncology \\ and Hematology, The Third People's Hospital of Shenzhen, Shenzhen, China; ${ }^{4}$ Department of Oncology, The First Affiliated Hospital of Ji Nan \\ University, Guangdong, China \\ Contributions: (I) Conception and design: L Xie, G Li; (II) Administrative support: L Xie, G Li; (III) Provision of study materials or patients: S \\ Qian, J An; (IV) Collection and assembly of data: Q Chen, X Liu, S Qian, J An; (V) Data analysis and interpretation: S Qian, J An, F Qi, L Ye; (VI) \\ Manuscript writing: All authors; (VII) Final approval of manuscript: All authors. \\ \#These authors contributed equally to this work. \\ Correspondence to: Lixin Xie. Center of Pulmonary and Critical Care Medicine, Chinese PLA General Hospital, Beijing, China. \\ Email: xielx301@126.com; Guobao Li. The Third People's Hospital of Shenzhen/National Clinical Research Center for Infectious Diseases, \\ Shenzhen, China. Email: feisanke-01@szsy.sustech.edu.cn.
}

Background: Coronavirus disease 2019 (COVID-19) has become a worldwide pandemic, affecting countries across the globe. With no current vaccine, treatment is still a critical intervention for minimizing morbidity and preventing disease-specific mortality. This study aimed to assess the clinical outcomes of critically ill COVID-19 patients using Tocilizumab treatment to provide recommendations for the treatment of COVID-19 patients with severe disease.

Methods: This was a retrospective analysis of medical records of six critically ill patients admitted to the Third People's Hospital of Shenzhen, China, from January 11 to February 26, 2020. Patient-related outcomes, including demographic, clinical, and laboratory characteristics before and after the initiation of Tocilizumab, were descriptively analyzed. Four to eight milligrams (mg)/kilogram (kg) of Tocilizumab was prescribed, with Chinese treatment guidelines.

Results: By the end of the last follow-up, Patient 1 and Patient 2 developed complications and died after using Tocilizumab for three to four days. Patient 4 died of multiple organ failure caused by cerebral infarction after using Tocilizumab for 39 days. Patient 3 and Patient 6 were discharged after 29 days and 33 days on Tocilizumab, respectively. Clinical symptoms, including fever, heart rate, and oxygen levels, improved after Tocilizumab use. Two patients appeared transient abnormal of liver or renal function indicator, and they can gradually recover. All elevated serum levels of inflammatory factors gradually decreased, except in Patient 2. Patient 3 and Patient 6's inflammatory lesions also significantly improved after initiating Tocilizumab.

Conclusions: Anti-inflammatory treatment with Tocilizumab was found to improve inflammatory responses in critically ill COVID-19 patients. Although some side reactions will occur, patients can gradually recover without affecting the efficacy of the therapy. However, the proper timing to start patients on Tocilizumab patients should be explored. Further prospective, randomized controlled clinical trials are called for.

Keywords: Coronavirus disease 2019 (COVID-19); inflammatory response; interleukin 6 (IL-6); Tocilizumab

Submitted May 27, 2020. Accepted for publication Jul 13, 2020.

doi: $10.21037 / \mathrm{atm}-20-5080$

View this article at: http://dx.doi.org/10.21037/atm-20-5080 


\section{Introduction}

In December 2019, a novel coronavirus, severe acute respiratory syndrome-coronavirus 2 (SARS-CoV-2), was discovered, causing a febrile respiratory illness later named the coronavirus disease 2019 (COVID-19) (1). As of May 1, 2020, the COVID-19 epidemic has spread to more than 200 countries and regions across the globe, infecting nearly over three million people and leading to more than 230,000 deaths (2). With reported data, most cases are classified as mild to moderate and found to be curable, with an overall low crude mortality rate (3). A proportion of patients, however, progress to severe disease characterized by respiratory dysfunction and often resulting in higher-related mortality (4). The ongoing spread of the disease is leading to overwhelmed healthcare systems, limiting the supplies of both protective equipment and supportive devices for healthcare workers and patients alike. With no vaccination developed yet, efforts to successfully treat these individuals and understand the factors leading to such progression are urgently needed.

Recent data has suggested that the SARS-CoV-2 virus can initiate a cytokine storm in infected patients, or a severe immune reaction releasing too many cytokines too quickly (5), and is closely related to the severity of the patient's disease (6). Interleukin 6 (IL-6) plays a vital role in this inflammatory storm (7), with data from one study showing that more than half of COVID-19 patients had elevated IL-6 levels upon admission (8), and increased IL-6 levels were associated with increased risk of death (9). Subsequently, inhibiting IL-6 has been found as a potential strategy to reduce the complications associated with COVID-19 infection (10). Few studies exist, however, which aim to understand and assess the impact of IL-6 inhibiting medications on disease outcomes of patients with severe COVID-19 (10). Tocilizumab, an IL-6R targeted monoclonal antibody $(\mathrm{mAb})$, received rapid approval in China to treat patients with severe COVID-19 (11). Thus, this study aimed to understand the epidemiological and clinical effects of Tocilizumab on a small cohort of patients with severe COVID-19 in Shenzhen, China.

We present the following article in accordance with the AME Case Series Checklist (available at http://dx.doi. org/10.21037/atm-20-5080).

\section{Methods}

\section{Study design and participants}

Our study was a retrospective, single-center clinical study conducted at the Third People's Hospital of Shenzhen, China. The Third People's Hospital of Shenzhen is the only government mandated hospital for the treatment of COVID-19 in Shenzhen. Patients who have diagnosed with the World Health Organization's (WHO) interim guidance (12) and identified as critically ill with acute respiratory distress syndrome (ARDS) on the Berlin 2012 criteria (13) were included. All procedures performed in this study involving human participants were in accordance with the Declaration of Helsinki (as revised in 2013). This study was approved by the ethics committee (No. 2020-021) of The Third People's Hospital of Shenzhen. All patients gave their oral consent to participate.

\section{Procedures}

Data on patients with pulmonary infection caused by SARS-CoV-2, which was confirmed by reverse transcription polymerase chain reaction (RT-PCR) testing (SARS$\mathrm{CoV}-2$ nucleic acid detection primers designed as following: Target1-ORF1ab Forward primer: CCC TGT TTT TAC ACT TAA, Reverse primer: ACG ATT GTG CAT CAG CTG A; Target2-N Forward primer: GGG GAA CTT CTC CTG CTA GAA T, Reverse primer: CAG ACA TTT TGC TCT CAA GCT G), clinical, and radiological assessments between January 11, 2020, and February 26, 2020, were collected.

The medical records of patients diagnosed with COVID-19 were collected from the Hospital Management Information System (HIS) and Laboratory Information System (LIS). A confirmed case was defined as having a positive result to high-throughput sequencing or real-time RT-PCR assay for nasal and pharyngeal swab specimens as tested by the local and national Chinese Centers for Disease Control and Prevention (China CDC) (1). All patients received computed tomography $(\mathrm{CT})$ examinations of the chest.

The following types of information were extracted: (I) demographic characteristics including age and sex, complications including ARDS, bacterial infection, fungal infections, septic shock, myocardial injury, liver/ renal function damage, and coagulation dysfunction; (II) epidemiological data, including travel history to the epidemic area (Hubei); (III) clinical symptoms, including temperature, and heart rate; (IV) oxygen levels: partial pressure/fractional inspired oxygen $\left(\mathrm{PaO}_{2} / \mathrm{FiO}_{2}\right) ;(\mathrm{V})$ laboratory examinations, including Interleukin-6(IL-6), C-reactive protein (CRP), Procalcitonin (PCT), lactate 
dehydrogenase (LDH), D-dimer, platelets(PLT), neutrophils counts (NEU), Troponin, Creatinine (CREA), urea nitrogen (UREA), alanine aminotransferase (ALT) and aspartate aminotransferase (AST) which are proxy measures to evaluate the safety and effectiveness of Tocilizumab; (VI) imaging, including CT of the chest; (VII) drug and oxygen treatments; and (VIII) outcomes, death or discharge. Missing data were supplemented through direct communication with attending physicians or other health care providers.

\section{Treatment schedule}

Many antiviral agents, antibiotics, and antifungal medications were provided to patients. Short-term, intravenous corticosteroid therapy was given to patients with severe dyspnea. All patients received continuous renal replacement therapy (CRRT) for dialysis. Patients were mechanically ventilated, depending on their level of hypoxemia. Some patients received re-infusion of plasma or antibodies from rehabilitation. All patients received the first dose of 320 milligrams (mg) of Tocilizumab, and a second dose could be administered at the physician's discretion. Data analysis was completed on April 25, 2020, when all patients completed at least twelve days of follow-up after Tocilizumab administration.

\section{Laboratory confirmation}

The serum levels of IL-6 [normal range 0-7 picograms per milliliter $(\mathrm{pg} / \mathrm{mL})$ ], C-Reactive Protein [normal range $<8 \mathrm{mg} /$ liter (L)], Procalcitonin [normal range $<0.1$ nanograms $(\mathrm{ng}) / \mathrm{mL}$, lactate dehydrogenase (normal range 120-250 U/L), D-dimer [normal range 0-0.5 microgram $(\mu \mathrm{g}) / \mathrm{mL}$ ], platelets (normal range $125-350 \times 10^{9} / \mathrm{L}$ ), neutrophils counts (normal range $1.8-6.3 \times 10^{9} / \mathrm{L}$ ), Troponin (normal range $<0.1 \mu \mathrm{g} / \mathrm{L}$ ), Creatinine [normal range 58-110 moles (umol)/L], urea nitrogen [normal range 3.6-9.5 millimole $(\mathrm{mmol}) / \mathrm{L}$ ], alanine aminotransferase (normal range 0-45 U/L) and aspartate aminotransferase (normal range 0-45 U/L) were observed before and after Tocilizumab administration on days 1, 2, 3, 5, 7, 9, and 12 .

\section{Results}

\section{Demographic and epidemiological characteristics of participants}

Six patients (five males and one female) with COVID-19 were treated with Tocilizumab and enrolled in the study. The median age of the six participants was 63.5 (range 59 to 69) years, and four had been to Hubei province. All six patients with concomitant chronic diseases received longterm treatment, and all patients had fever upon admission, while half had hypertension (Table 1).

\section{Patients' response to treatment with Tocilizumab}

Before receiving Tocilizumab, six patients received invasive ventilator maintenance treatment. Patient 1 and Patient 2 received extracorporeal membrane oxygenation (ECMO) for 11 and 17 days, respectively (Figure 1). All six patients were treated with antiviral, anti-infective, and glucocorticoid therapies. Other treatment mechanisms were used as well, including transfusion with plasma and CRRT (Table 1).

The median time from using invasive mechanical ventilation to use of Tocilizumab was 14.5 (range 1 to 21) days. Patient 1 and Patient 2 died of multiple organ failure from COVID-19 on Day 3 and Day 4, respectively, after using Tocilizumab. Patient 5 died of cerebral infarction, but not SARS-CoV-2, after 39 days on Tocilizumab. Patient 3 and Patient 6 were discharged after 29 and 33 days on Tocilizumab, respectively, and Patient 4 had to receive invasive ventilator maintenance treatment (Table 1, Figure 1). All six patients received the first dose of $320 \mathrm{mg}$ as their first dose of Tocilizumab, while Patient 3 received a second dose of $240 \mathrm{mg}$, and Patient 4 received a second dose of $640 \mathrm{mg}$.

\section{Clinical response to using Tocilizumab}

Four patients (Patients 1, 4, 5, 6) had a fever (armpit temperature $>37^{\circ} \mathrm{C}$ ) before medication, but this returned to normal for all patients after initiation of treatment (Table 2). Four patients also experienced an elevated heart rate ( $>100$ beats/minute), but this too returned to normal at the end of observation (Figure 2). All six patients were diagnosed with ARDS before taking the drug. After taking Tocilizumab, only Patients 1 and 2 had no improvement in their breathing and eventually died. The remaining four patients' oxygenation levels gradually increased, however Patient 4 still experienced mild ARDS after treatment (Figure 2). Thus, after taking Tocilizumab, the patients' clinical symptoms and respiratory states improved.

\section{Laboratory response to the use of Tocilizumab}

All six patients had higher levels of IL-6 (normal range 
Table 1 Baseline demographic and clinical characteristics of six critically ill COVID-19 patients from the Third People's Hospital of Shenzhen, China

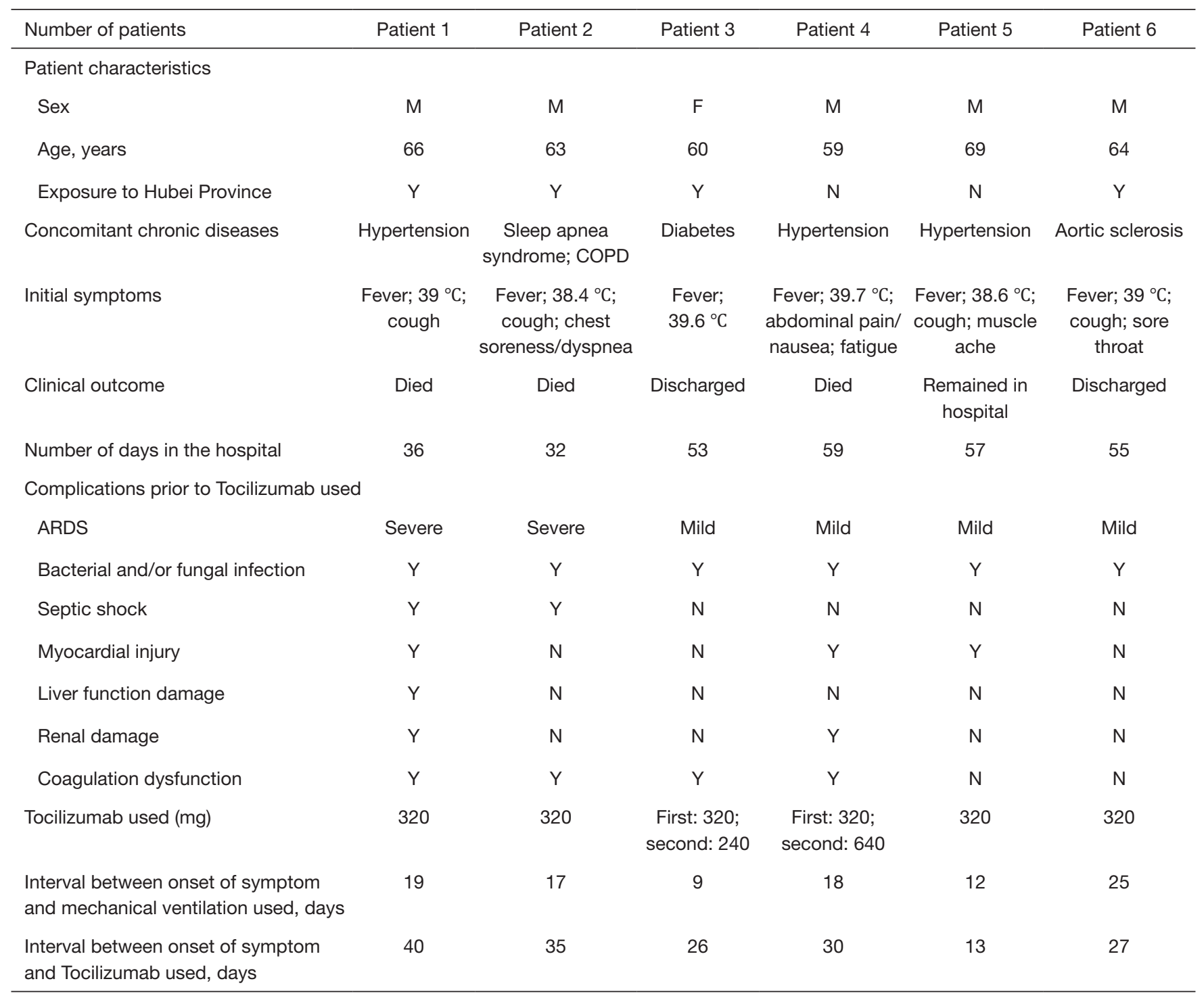

M, male; F, female; Y, yes; N, no; mg, milligrams; C, celsius; COPD, chronic obstructive pulmonary disease; ARDS, acute respiratory distress syndrome.

0-7 pg/mL) before treatment with Tocilizumab. Serum IL-6 levels in five patients decreased (Patient 1: 1,449.0 vs. 944.6; Patient 3: 711.0 vs. 50.5; Patient 4: 1,679.0 vs. 381.9; Patient 5: 438.2 vs. 27.0; Patient 6: 797.9 vs. 47.6) after initiating Tocilizumab (Figure 3). Only Patient 2's levels of IL-6 did not change while on Tocilizumab $(5,000$ vs. 5,000) (Figure 3), and the patient subsequently died on day four after initiating treatment. CRP serum levels in all patients were higher than the normal range $(<8 \mathrm{mg} / \mathrm{L})$ before Tocilizumab medication and showed a decline trend after
Tocilizumab was used (Patient 1: 236.89 vs. 93.27; Patient 2: 281.55 vs. 130.47; Patient 3: 176.48 vs. 0.70; Patient 4: 213.68 vs. 49.25; Patient 5: 242.67 vs. 19.09; Patient 6: 233.70 vs. 3.57) (Figure 3). The PCT serum levels of five patients was abnormally elevated (normal $<0.1 \mathrm{ng} / \mathrm{mL}$ ) before initiating medication but decreased after the application of the Tocilizumab (Patient 1: 86.36 vs. 48.22; Patient 2: 100 vs. 34.16; Patient 3: 0.13 vs. 0.03; Patient 4: 0.96 vs. 0.40; Patient 5: 7.34 vs. 0.17; Patient 6: 1.30 vs. 0.06) (Figure 3). The D-dimer level in all six patients 


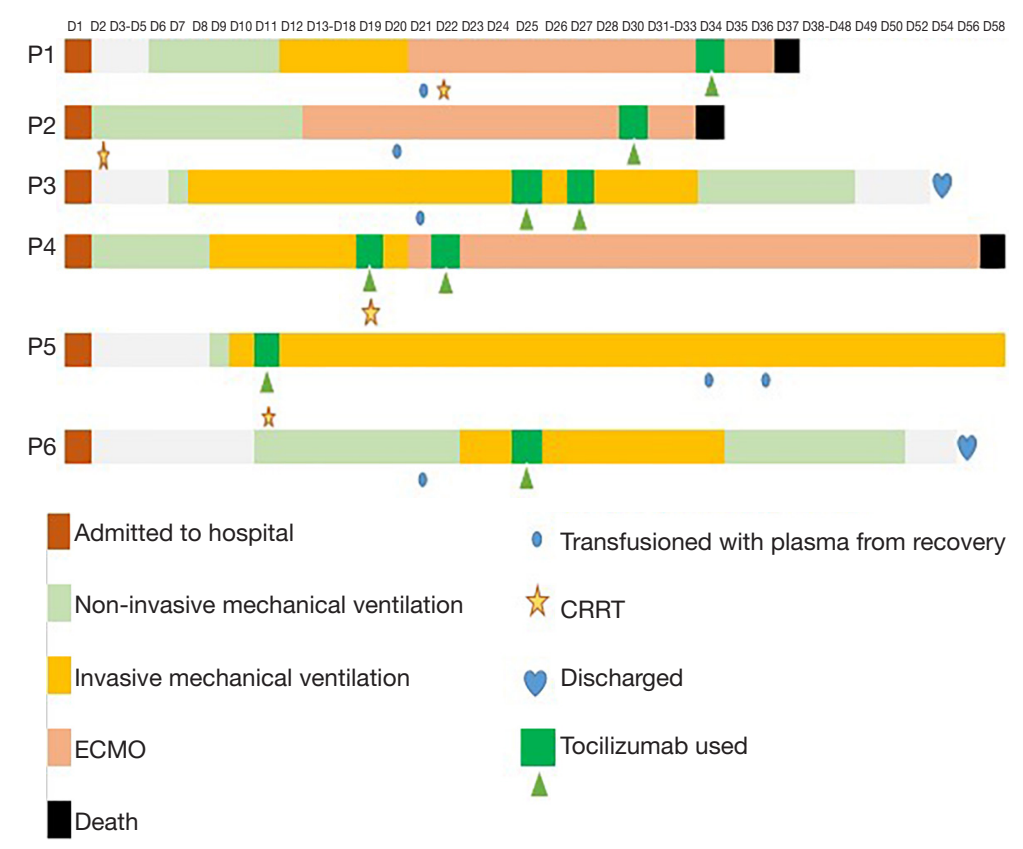

Figure 1 The timeline from admission, to initiation of treatment, and patient-related outcome of six critically ill COVID-10 patients from the Third People's Hospital of Shenzhen, China. Figure 1 summarizes the timeline from admission to patient-related outcomes among the six critically ill patients enrolled in the study. The orange box is the time at which the patients were admitted to the hospital (day 1 ). All patients required non-invasive medical ventilation at some point throughout their hospital admission, as showed by the light green boxes. All patients except Patient 2, however, required invasive mechanical ventilation, as represented by the yellow boxes. Patients started Tocilizumab at different points throughout the study period, as showed by the dark green boxes. Three patients in the study died (black boxes), two patients were discharged (blue hearts), and one patient was still admitted to the hospital at the time of publication. CRRT, continuous renal replacement therapy; ECMO, extracorporeal membrane oxygenation.

was abnormally increased before starting the Tocilizumab application. However, these levels also dropped after starting the drug (Figure 3).

Except for Patients 3 and 6 who experienced an increase in PLT after taking the medication, the remaining four patients had lower than normal PLT levels. All patients showed an increase in numbers of neutrophils before and after treatment, and none had experienced neutropenia.

All six patients showed elevated LDH status before and after medication. Although some patients showed an increase and fluctuation after treatment (Patient 3 and 4), all six patients eventually showed a downward trend in LDH levels, but LDH levels did not return to normal in any of the 6 patients after treatment (Table 2).

Three patients (Patients 1, 4, and 5) had elevated Troponin levels (normal $<0.1 \mu \mathrm{g} / \mathrm{L}$ ) before starting Tocilizumab, indicating myocardial injury, but these levels dropped after initiating treatment (Table 2).

Patient 5 experienced an increase in both CREA and
UREA before taking Tocilizumab, which remained high even after initiation. Patient 2 experienced similar increasing levels of UREA before and after treatment initiation (Table 2).

Liver enzymes among the patients remained stable, except in Patient 1, who experienced abnormally elevated liver enzymes before medication, though this declined after treatment. Patient 4 had normal liver enzymes before medication, but experienced a sharp increase after medication, with the highest levels reported on the 4th day after medication (ALT 3,947 U/L, AST 6,354 U/L), which gradually declined, however. While AST levels returned to normal, ALT remained high, which could be a side effect of Tocilizumab.

\section{CT images after use of Tocilizumab}

All patients had typical symptoms of bilateral pneumonia before medication. The CT imaging of Patient 3 and 
Table 2 Clinical symptoms before and after use of Tocilizumab

\begin{tabular}{|c|c|c|c|c|c|c|c|c|c|c|c|}
\hline Patient & Before TCZ & After TCZ(d1) & $\mathrm{d} 2$ & d3 & $\mathrm{d} 4$ & d5 & d6 & $\mathrm{d} 7$ & d9 & d12 & Clinical outcome \\
\hline Patient 1 & $65 \downarrow$ & $81 \downarrow$ & $110 \downarrow$ & Died & & & & & & & Died \\
\hline Patient 2 & $67 \downarrow$ & $36 \downarrow$ & $25 \downarrow$ & $20 \downarrow$ & Died & & & & & & Died \\
\hline Patient 3 & 345 & $378 \uparrow$ & $376 \uparrow$ & $396 \uparrow$ & $419 \uparrow$ & $417 \uparrow$ & $377 \uparrow$ & $364 \uparrow$ & 294 & 287 & Discharged \\
\hline Patient 5 & 171 & 139 & 126 & $113 \downarrow$ & $105 \downarrow$ & $107 \downarrow$ & $102 \downarrow$ & $102 \downarrow$ & $95 \downarrow$ & $\mathrm{N}$ & Remained in hospital \\
\hline Patient 6 & 324 & 297 & $354 \uparrow$ & $358 \uparrow$ & $384 \uparrow$ & $360 \uparrow$ & $373 \uparrow$ & 336 & 319 & 289 & Discharged \\
\hline \multicolumn{12}{|c|}{ NEUT (NR 1.8-6.3) ×10 $/ \mathrm{L}$} \\
\hline Patient 1 & $25.5 \uparrow$ & $19.9 \uparrow$ & $17.5 \uparrow$ & Die & & & & & & & Died \\
\hline Patient 4 & 3.9 & 5.3 & 3.3 & 3.7 & $7.8 \uparrow$ & 6.3 & 6.1 & 4.9 & 5.2 & 6.2 & Died \\
\hline Patient 5 & $10.9 \uparrow$ & $7.1 \uparrow$ & $8.8 \uparrow$ & 6.0 & $7.6 \uparrow$ & $8.2 \uparrow$ & $9.1 \uparrow$ & $11.9 \uparrow$ & $10.8 \uparrow$ & $6.9 \uparrow$ & Remained in hospital \\
\hline Patient 6 & $7.0 \uparrow$ & 4.4 & 3.6 & 3.9 & 5.9 & $10.4 \uparrow$ & $18.6 \uparrow$ & $13.8 \uparrow$ & $9.8 \uparrow$ & 5.8 & Discharged \\
\hline \multicolumn{12}{|c|}{ Tnl Ultra (NR <0.1 $\mu \mathrm{g} / \mathrm{L})$} \\
\hline Patient 1 & $2.29 \uparrow$ & NA & $0.65 \uparrow$ & Die & & & & & & & Died \\
\hline Patient 2 & NA & NA & NA & NA & Die & & & & & & Died \\
\hline Patient 3 & $<0.012$ & NA & NA & NA & NA & NA & $0.02 \uparrow$ & $<0.012$ & $<0.012$ & $<0.012$ & Discharged \\
\hline Patient 4 & $0.34 \uparrow$ & $0.11 \uparrow$ & $0.10 \uparrow$ & $1.80 \uparrow$ & NA & $0.90 \uparrow$ & $0.50 \uparrow$ & NA & $0.20 \uparrow$ & $<0.012$ & Died \\
\hline Patient 3 & $37.6 \downarrow$ & $53.7 \downarrow$ & $43.7 \downarrow$ & $35.2 \downarrow$ & $47.5 \downarrow$ & $47.8 \downarrow$ & $41.9 \downarrow$ & $38.4 \downarrow$ & $33.9 \downarrow$ & $36.1 \downarrow$ & Discharged \\
\hline Patient 4 & $119.5 \uparrow$ & $54.0 \downarrow$ & $57.0 \downarrow$ & $41.0 \downarrow$ & 71.0 & 81.0 & 83.0 & 79.0 & 67.0 & 81.0 & Died \\
\hline Patient 5 & $177.0 \uparrow$ & $273.0 \uparrow$ & $264.0 \uparrow$ & $184.0 \uparrow$ & $189.0 \uparrow$ & $201.0 \uparrow$ & $179.0 \uparrow$ & $179.0 \uparrow$ & $204.0 \uparrow$ & $181.0 \uparrow$ & Remained in hospital \\
\hline Patient 6 & 80.0 & 77.0 & 83.6 & 87.9 & 105.3 & $114.5 \uparrow$ & 81.4 & 74.5 & 64.0 & 57.0 & Discharged \\
\hline \multicolumn{12}{|c|}{ UREA (NR 3.6-9.5 mmol/L) } \\
\hline Patient 1 & 9.5 & 9.5 & 9.1 & Die & & & & & & & Died \\
\hline Patient 2 & $9.8 \uparrow$ & $13.0 \uparrow$ & $13.0 \uparrow$ & $14.1 \uparrow$ & Die & & & & & & Died \\
\hline Patient 3 & 4.8 & 4.5 & 5.9 & 4.3 & 3.7 & 3.8 & 4.9 & 4.8 & 6.7 & 5.3 & Discharged \\
\hline Patient 4 & 5.6 & 3.5 & 2.7 & 4.2 & 5.9 & 5.5 & 6.9 & 7.7 & 7.8 & 6.7 & Died \\
\hline Patient 5 & $12.7 \uparrow$ & $14.6 \uparrow$ & $13.7 \uparrow$ & $11.3 \uparrow$ & $12.4 \uparrow$ & $16.2 \uparrow$ & $16.9 \uparrow$ & $19.3 \uparrow$ & $23.9 \uparrow$ & $14.4 \uparrow$ & Remained in hospital \\
\hline Patient 6 & 3.7 & 6.9 & 6.9 & 7.6 & $12.0 \uparrow$ & $14.0 \uparrow$ & $11.6 \uparrow$ & $9.7 \uparrow$ & 7.9 & 8.2 & Discharged \\
\hline
\end{tabular}

Table 2 (continued) 
Table 2 (continued)

\begin{tabular}{|c|c|c|c|c|c|c|c|c|c|c|c|}
\hline Patient & Before TCZ & fter TCZ(d1) & $\mathrm{d} 2$ & d3 & $\mathrm{d} 4$ & d5 & d6 & $\mathrm{d} 7$ & d9 & d12 & Clinical outcome \\
\hline Patient 1 & $476.0 \uparrow$ & $471.0 \uparrow$ & $310.0 \uparrow$ & Die & & & & & & & Died \\
\hline Patient 2 & 16.0 & 16.0 & 29.0 & 28.0 & Die & & & & & & Died \\
\hline Patient 3 & 30.5 & 28.4 & 41.0 & 31.2 & 39.0 & 38.8 & 32.4 & 23.2 & 20.3 & 18.8 & Discharged \\
\hline Patient 5 & 28.0 & $\mathrm{~N}$ & 37.0 & 59.0 & 52.0 & 50.0 & 38.0 & 30.0 & 28.0 & 42.0 & Remained in hospital \\
\hline Patient 6 & 19.0 & $\mathrm{~N}$ & $\mathrm{~N}$ & $\mathrm{~N}$ & $\mathrm{~N}$ & $\mathrm{~N}$ & $\mathrm{~N}$ & $\mathrm{~N}$ & 48.0 & 44.0 & Discharged \\
\hline \multicolumn{12}{|c|}{ AST (NR 0-45 U/L) } \\
\hline Patient 1 & $979.0 \uparrow$ & $652.0 \uparrow$ & $461.0 \uparrow$ & Die & & & & & & & Died \\
\hline Patient 4 & 44.0 & 48.0 & $162.0 \uparrow$ & $2,034.0 \uparrow$ & $6,354.0 \uparrow$ & $2,952.0 \uparrow$ & $859.0 \uparrow$ & $67.0 \uparrow$ & $64.0 \uparrow$ & 37.0 & Died \\
\hline Patient 5 & 45.0 & & 47.0 & $66.0 \uparrow$ & $80.0 \uparrow$ & $54.0 \uparrow$ & 36.0 & 36.0 & 29.0 & 33.0 & Remained in hospital \\
\hline Patient 6 & 19.0 & $\mathrm{~N}$ & $\mathrm{~N}$ & $\mathrm{~N}$ & $\mathrm{~N}$ & $\mathrm{~N}$ & $\mathrm{~N}$ & $\mathrm{~N}$ & 39.0 & 38.0 & Discharged \\
\hline
\end{tabular}

TCZ, Tocilizumab; d1-d12 means days after TCZ, NA, not available; NR, normal range; " $\uparrow$ "showed the Index above normal; " $\downarrow$ " showed the Index below normal.
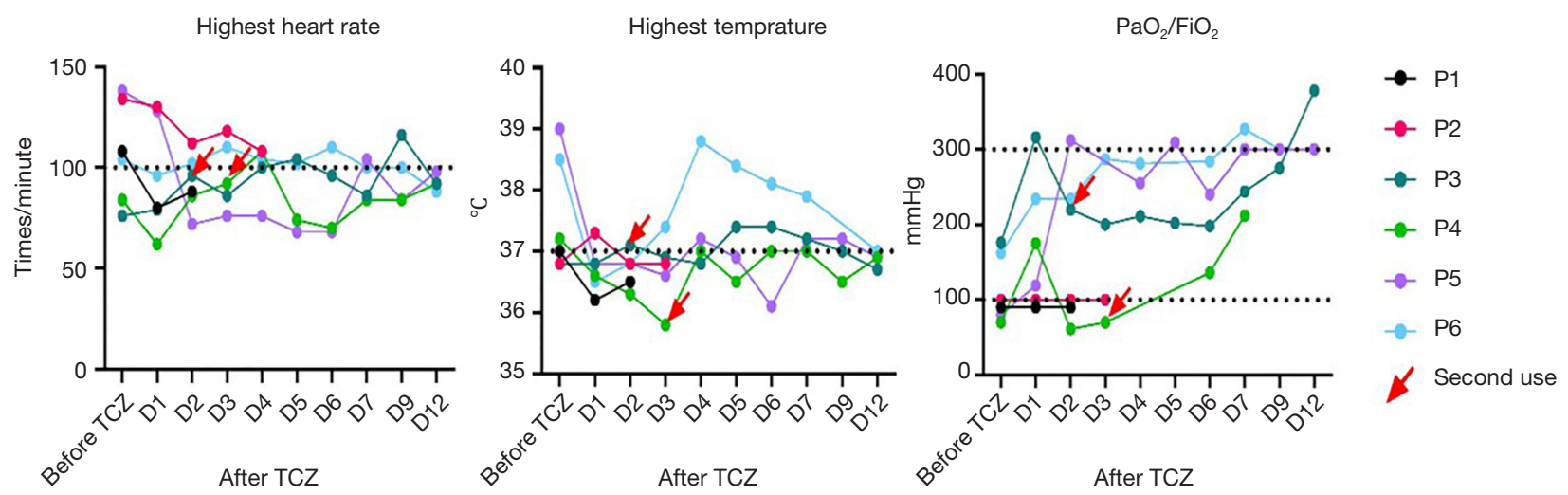

Figure 2 The change of clinical symptoms and oxygenation index before and after medication of Tocilizumab. The figures show the changing trends of the highest heart rate, temperature, $\mathrm{PaO}_{2} / \mathrm{FiO}_{2}$ before and after the application of Tocilizumab. Although there are some fluctuations after taking the drug, all indicators improved over time. $\mathrm{PaO}_{2} / \mathrm{FiO}_{2}$, partial pressure/fractional inspired oxygen; TCZ, Tocilizumab.

Patient 6 was reviewed about 1 week after medication, showing reduced inflammatory lesions (Figure 4). Patient 1 died three days after starting treatment, while Patient 2 died four days after. Patients 4 and 5 were placed on continuous mechanical ventilation, so they did not have a follow-up CT scan.

\section{Discussion}

COVID-19 is quickly becoming an international public health threat, leading to pulmonary infection, coagulopathy, sepsis, persistent hypoxemia, and organ damage in critically ill patients (4). As no medical intervention, including 
IL6

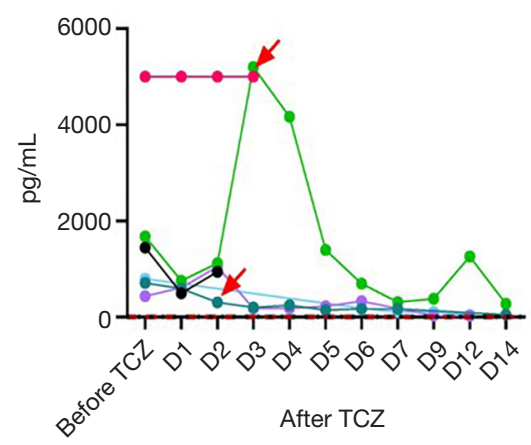

PCT

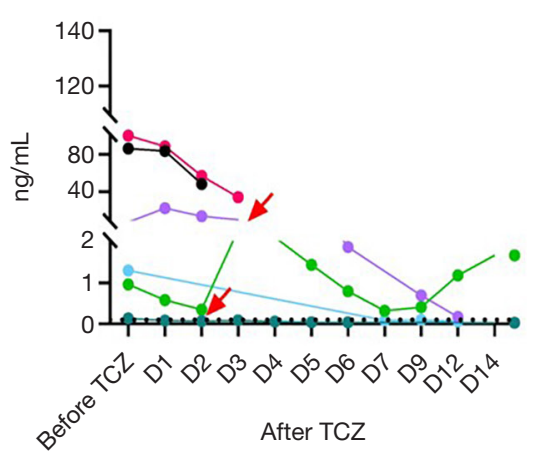

CRP

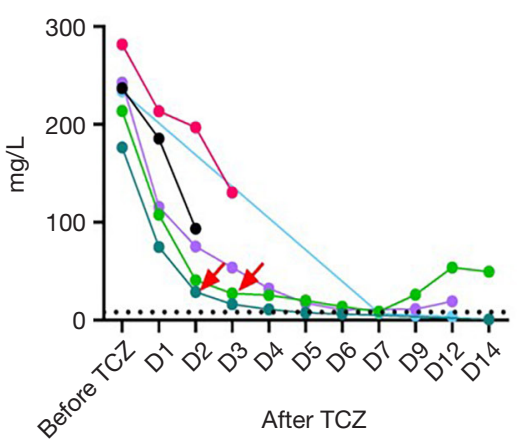

LDH

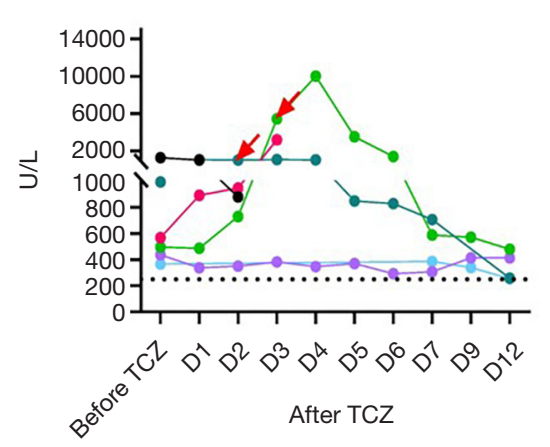

Figure 3 Changes in laboratory indicators before and after initiation of Tocilizumab among six critically ill COVID-19 Patients at the Third People's Hospital of Shenzhen. The figures show the changing trends of IL-6, CRP, PCT, and D-dimer before and after the application of Tocilizumab. Although there are some fluctuations after taking the drug, all indicators decrease over time. IL-6, interleukin 6; CRP, C-reactive protein; PCT, Procalcitonin; pg/ML, pictogram/milliliter; mg/L, milligram/liter; ng/L, nanogram/liter; ug/mL, microgram/liter.

a vaccine, which can prevent COVID-19 at this time, determining an effective and safe treatment strategy for patients is adamant (14). This study aimed to assess the impact of using Tocilizumab on patient-related outcomes, the results of which can be used to streamline treatment decisions better and improve patient health.

Among the six critically ill patients in this cohort, all had a severe respiratory infection requiring mechanical ventilation support. Several studies have observed the potential role and value of anti-inflammatory strategies in the treatment of COVID-19, but few related clinical studies assessed the impact of Tocilizumab, specifically. A clinical trial of Tocilizumab found that all 21 patients enrolled in the study improved after treatment, though only two patients were categorized as having severe disease (15). Similarly, another single-center study (16) reported that of 15 patients, ten showed decreasing levels of IL-6 after taking the medication. More so, of four patients who failed treatment, IL-6 continued to increase after medication, which was consistent with Patient 2 in this study. Our study also found that all six patients had improved clinical symptoms during the observation period after starting therapy, including reduced fever and improved heart rate and oxygen levels. The CT images of the lungs of the two patients discharged were improved. Thus, our study further builds upon these findings, highlighting the potential efficacy of using such therapy in those who may be sickest.

IL-6R may inhibit the receptor-mediated clearance pathway, leading to an accumulation of IL-6 in patients' serum (17). Subsequently, Tocilizumab may not directly act on immune cells to inhibit IL-6 production. IL-6 inhibitors play a crucial role in curbing the inflammatory response, which may help inhibit the development of sepsis and the continuous deterioration of organ function damage (16). 


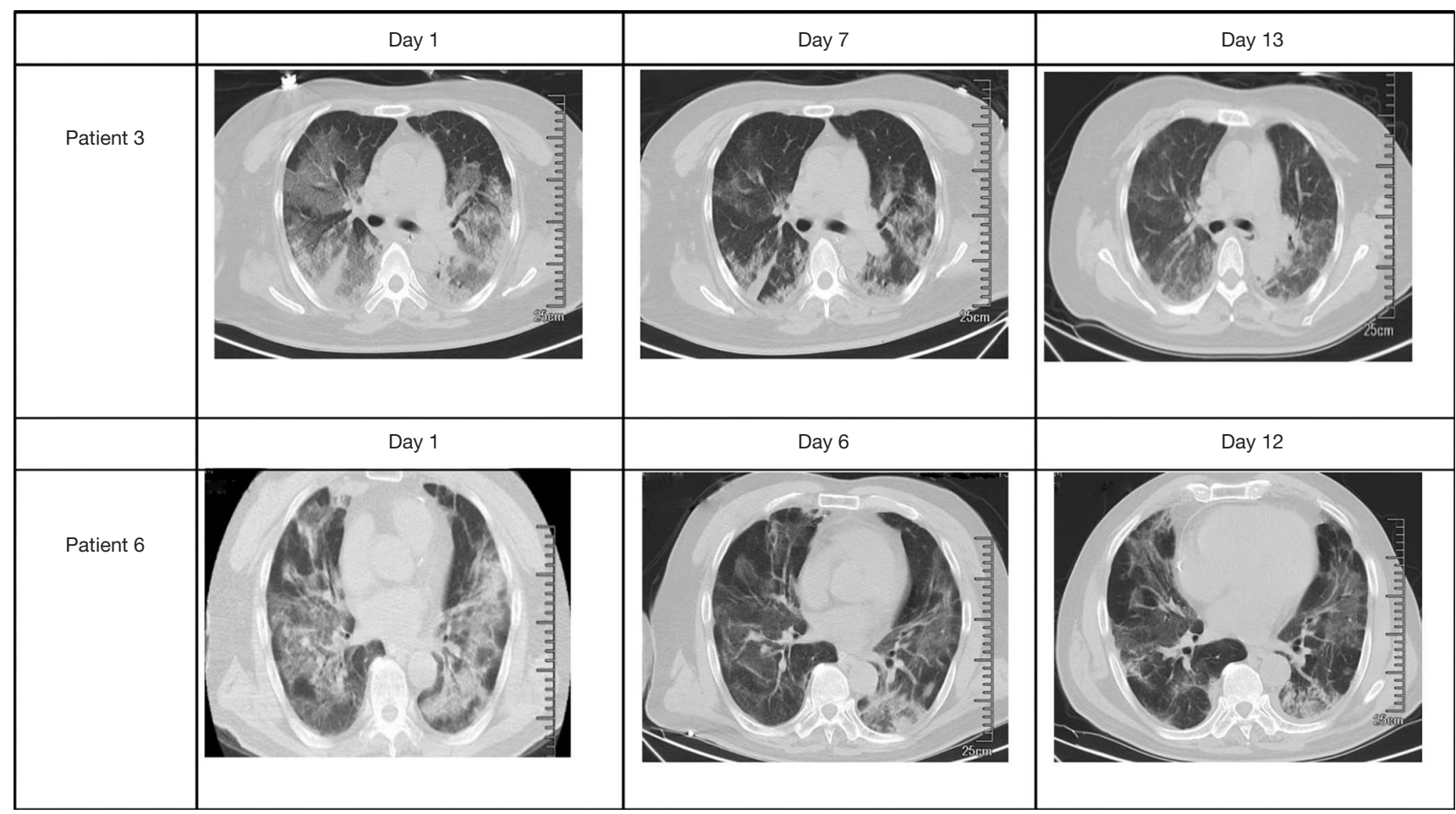

Figure 4 Chest computed tomography (CT) changes in two critically ill COVID-19 patients before and after Tocilizumab. Only two patients underwent CT examination before and after medication. For Patient 3, the first chest CT scan showed significant ground-glass opacity on both lungs one day after the use of Tocilizumab. On days 7 and 13 of Tocilizumab use, the lung lesions on the chest CT imaging were significantly reduced, accompanied by improved clinical conditions. For Patient 6 , the first chest CT scan showed significant groundglass opacity on both lungs one day after the use of Tocilizumab. At days 6 and 12, the lung lesions on the chest CT scan were significantly reduced, accompanied by improved clinical conditions. Black arrow marked the location of inflammatory lesions.

Also, earlier observations found that the levels of various inflammatory factors other than IL-6 in the alveolar lavage fluid of critically ill patients fluctuated (18). It is difficult to block the IL-6 signaling pathway alone effectively. For example, in this study, Patient 5 continued to show aggravated inflammation of the lungs, despite the decrease in IL-6 levels after taking Tocilizumab. Specifically, the patient's CT showed that bilateral lung inflammation increased, showing that other inflammatory factors persisted $(1,19)$. Therefore, it may be more meaningful to explore the critical mechanisms of inflammatory injury and use a multi-therapeutic approach to block multiple inflammatorycausing factors for the treatment of critically ill COVID-19 patients.

In this study, patients who received Tocilizumab earlier in the course of their infection had better outcomes compared to more severe cases. Other studies have shown that COVID-19 may cause infection of the nervous system
$(20,21)$, which could explain these poorer outcomes. In our study, Patient 4, who eventually died of cerebral infarction, unfortunately, however, the patient did not have a lumbar puncture examination to determine whether there was SARS-CoV-2 present in the cerebrospinal fluid.

Additionally, as observed in this study, not only did IL-6 decrease after treatment among the patients in this study, but other indicators, including CRP, PCT, D-Dimer, and $\mathrm{LDH}$ that reflect severe disease, also decreased. It may be more helpful to use Tocilizumab in the early stages of inflammation when inflammatory factors fluctuate significantly.

Early blocking of the IL-6 signaling pathway, however, may also affect viral clearance, as some studies have found that IL-6 may play an essential role in the initial immune response after viral infection by promoting neutrophilmediated virus clearance. In one animal model, IL-6/IL-6R deficiency was found to lead to the persistence of influenza 
virus infection and eventually lead to the death of mice (22). Also, some studies believe that continuous IL-6 elevation may impact the efficacy of Tocilizumab (16), including observed in part of this cohort of critically ill patients. Extended clinical observations to determine the optimal timing and dosage of Tocilizumab for reducing levels of inflammation in severe patients could help bolster existing treatment strategies and improve patient outcomes in the future.

Lastly, after taking the drug, all six patients did not experience high fever, chills, high blood pressure, and their mechanical ventilation mode was not changed significantly. Four patients had decreased levels of PLT. One patient had abnormal renal function indicators. Another patient had abnormal liver enzymes. However, these abnormalities may not be related to the side effects of using Tocilizumab. These six patients were all seriously ill during medication. They can exhibit the above abnormalities due to SARSCoV-2. Therefore, it is difficult to explain whether the decrease in PLT, abnormal renal function, and liver enzymes are caused by drug side effects or disease itself.

This study is not without deficiencies. First, this is a retrospective clinical observation from a singlecenter, with a few cases and a brief observation period. However, the Third People's Hospital in Shenzhen is the only government-approved facility for the treatment of COVID-19 patients. Thus, our sample could be representative of other patients in the region. A more extensive, protective analysis considering multiple therapies would confirm the findings of this study. Second, this study lacked dynamic and detailed viral nucleic acid load testing results and the changing levels of inflammatory factors in alveolar lavage fluid.

Further exploration of the underlying pathophysiological mechanism of inflammatory factors is needed. Lastly, the pathophysiological changes of critically ill patients are complicated, and the treatment and intervention methods are diverse. The potential value of Tocilizumab as an antiinflammatory strategy still requires large-scale, randomized clinical trials.

\section{Conclusions}

Reasonable application of Tocilizumab can improve clinical symptoms and exert anti-inflammatory of critically ill COVID-19 patients. Although some side reactions will occur, patients can gradually recover without affecting the efficacy of the therapy, but the proper timing, of treatment, should be explored. Further controlled trials assessing the efficacy of Tocilizumab should be considered.

\section{Acknowledgments}

Thanks are due to Professor Fu-sheng Wang for editing this paper. This paper was polished English language by Professor Sarah Robbins Scott on behalf of the Bill \& Melinda Gates Foundation.

Funding: This research was funded by the Medical and Health Three Famous Projects in Shenzhen and awarded to Professor Liu You-Ning, General Hospital of the Chinese People's Liberation Army, Respiratory System Critical Illness, and Major Emerging Infectious Diseases Diagnosis and Research Team (SZSM201612025). This research was funded by the 2020 Guangdong Province Special Project on Emergency Research on Prevention and Control of New Coronavirus Infection Technology (2020B1111340030). China National Key Research Program (2018ZX09201013); China PLA Key Research Program (A3704041902-03); Research Foundation of Medical Science and Technology of Guangdong Province (No. B2019132); Ji Nan University Central University Basic Research Fund (No. 21619359).

\section{Footnote}

Reporting Checklist: The authors have completed the AME Case Series Checklist. Available at http://dx.doi. org/10.21037/atm-20-5080

Data Sharing Statement: Available at http://dx.doi. org/10.21037/atm-20-5080

Conflicts of Interest: All authors have completed the ICMJE uniform disclosure form (available at http://dx.doi. org/10.21037/atm-20-5080). The authors have no conflicts of interest to declare.

Ethical Statement: The authors are accountable for all aspects of the work in ensuring that questions related to the accuracy or integrity of any part of the work are appropriately investigated and resolved. All procedures performed in this study involving human participants were in accordance with the Declaration of Helsinki (as revised in 2013). This study was approved by the ethics committee (No. 2020-021) of The Third People's Hospital of Shenzhen. All patients gave their oral consent to participate. 
Open Access Statement: This is an Open Access article distributed in accordance with the Creative Commons Attribution-NonCommercial-NoDerivs 4.0 International License (CC BY-NC-ND 4.0), which permits the noncommercial replication and distribution of the article with the strict proviso that no changes or edits are made and the original work is properly cited (including links to both the formal publication through the relevant DOI and the license). See: https://creativecommons.org/licenses/by-ncnd/4.0/.

\section{References}

1. Huang C, Wang Y, Li X, et al. Clinical features of patients infected with 2019 novel coronavirus in Wuhan, China. Lancet 2020;395:497-506.

2. Available online: https://www.who.int/docs/default-source/ coronaviruse/situation-reports/20200501-covid-19-sitrep. pdf?sfursn=742f4a18_2

3. Guan WJ, Ni ZY, Hu Y, et al. Clinical Characteristics of Coronavirus Disease 2019 in China. N Engl J Med 2020;382:1708-20.

4. Yang $\mathrm{X}, \mathrm{Yu} \mathrm{Y}, \mathrm{Xu} \mathrm{J}$, et al. Clinical course and outcomes of critically ill patients with SARS-CoV-2 pneumonia in Wuhan, China: a single-centered, retrospective, observational study. Lancet Respir Med 2020;8:475-81.

5. Mehta P, McAuley DF, Brown M, et al. COVID-19: consider cytokine storm syndromes and immunosuppression. Lancet 2020;395:1033-4.

6. Eric Anthony Coomes, Hourmazd Haghbayan. Interleukin-6 in COVID-19: A Systematic Review and Meta-Analysis. medRxiv 2020. doi: https://doi. org/10.1101/2020.03.3020048058.

7. Tanaka T, Narazaki M, Kishimoto T. Immunotherapeutic implications of IL-6 blockade for cytokine storm. Immunotherapy 2016;8:959-70.

8. Chen N, Zhou M, Dong X, et al. Epidemiological and clinical characteristics of 99 cases of 2019 novel coronavirus pneumonia in Wuhan, China: a descriptive study. Lancet 2020;395:507-13.

9. Wu C, Chen X, Cai Y, et al. Risk Factors Associated With Acute Respiratory Distress Syndrome and Death in Patients With Coronavirus Disease 2019 Pneumonia in Wuhan, China. JAMA Intern Med 2020. [Epub ahead of print].

10. Aziz M, Fatima R, Assaly R. Elevated Interleukin-6 and Severe COVID-19: A Meta-Analysis. J Med Virol 2020. [Epub ahead of print].
11. National Health Commission of China, 2020.

12. WHO. Clinical management of severe acute respiratory infection when Novel coronavirus ( $\mathrm{nCoV}$ ) infection is suspected: interim guidance. Available online: https://www. who.int/internal

13. ARDS Definition Task Force, Ranieri VM, Rubenfeld GD. Acute respiratory distress syndrome: the Berlin Definition. JAMA 2012;307:2526-33.

14. Cao X. COVID-19: immunopathology and its implications for therapy. Nat Rev Immunol 2020;20:269-70.

15. Xu X, Han M, Li T, et al. Effective Treatment of Severe COVID-19 Patients with Tocilizumab. Proc Natl Acad Sci U S A 2020;117:10970-5.

16. Luo P, Liu Y, Qiu L, et al. Tocilizumab treatment in COVID-19: A single center experience. J Med Virol 2020;92:814-8.

17. Nishimoto N, Terao K, Mima T, et al. Mechanisms and pathologic significances in increase in serum interleukin-6 (IL-6) and soluble IL-6 receptor after administration of an anti-IL-6 receptor antibody, tocilizumab, in patients with rheumatoid arthritis and Castleman disease. Blood 2008;112:3959-64.

18. Xu G, Liu Y, Li H, et al. Dissecting the human immune system with single cell RNA sequencing technology. J Leukoc Biol 2020;107:613-23.

19. Liu J, Li S, Liu J, et al. Longitudinal characteristics of lymphocyte responses and cytokine profiles in the peripheral blood of SARS-CoV-2 infected patients. medRxiv 2020. doi: 10.1101/2020.02.16 20023671.

20. Li YC, Bai WZ, Hashikawa T. The neuroinvasive potential of SARS-CoV2 may be at least partially responsible for the respiratory failure of COVID-19 patients. J Med Virol 2020;92:552-5.

21. Zhou L, Zhang M, Wang J, Gao J. Sars-Cov-2: underestimated damage to nervous system. Travel Med Infect Dis 2020. [Epub ahead of print].

22. Dienz O, Rud JG, Eaton SM, et al. Essential role of IL-6 in protection against $\mathrm{H} 1 \mathrm{~N} 1$ influenza virus by promoting neutrophil survival in the lung. Mucosal Immunol 2012;5:258-66.

(English Language Editor: J. Chapnick)

Cite this article as: Qian S, An J, Qi F, Ye L, Chen Q, Liu X, Xie L, Li G. Tocilizumab exerts anti-inflammatory activity in six critically ill COVID-19 patients: a retrospective analysis. Ann Transl Med 2020;8(14):881. doi: 10.21037/atm-20-5080 